\title{
Sample concentration and buffer exchange utilizing a miniaturized tangential flow filtration (TFF) system \\ Logan, Baker; Rodionov, Dmitry
}

Concentration and buffer exchange steps are integral to any preparative scale protein production, whether for structural biology, or general biochemistry needs. These steps must be done at least once at the end of the purification protocol, or even between different chromatographic steps, adding up to a significant amount of time.

Ultrafiltration is the current standard for concentrating proteins and, on lab scale, typically involves disposable centrifuge-driven devices. These devices are dead-end in nature (the flow is perpendicular to the membrane) and create a concentration gradient at the membrane. The concentration at the membrane can far exceed the target slowing down the flow of the filtrate and increasing material loss due to aggregation/precipitation. The only way to monitor the concentration progress or to mix the sample is by periodic interruption of the centrifugation, making it a fairly hands-on method.

Membrane dialysis is the most popular buffer exchange method also involving a molecular weight cutoff membrane driven by the osmotic pressure. While being a hands-off method, it requires a large excess of the dialysis buffer, a long dialysis time (8-12 hours) and a subsequent concentration step.

On industrial scale, both buffer exchange and concentration are accomplished with a single device employing tangential flow filtration (TFF). In addition to the filtrate flow across the membrane, TFF systems will also have an active flow parallel (or tangential) to the membrane. This flow mixes the sample (retentate) in real time, drastically reducing the concentration gradient and allowing for filter dialysis (diafiltration) if a desired buffer was added to the retentate. However, even the smallest of the TFF devices do not fit in the typical lab workflow having the dead volume in tens to hundreds of milliliters.

Here we present our miniaturized TFF system, one of the smallest device of its kind available, intended specifically for lab scale purifications. It features a consumable cartridge that encapsulates a microfluidic pump and a slightly larger membrane than a typical $15 \mathrm{~mL}$ centrifugal device $\left(\sim 7.1 \mathrm{~cm}^{\wedge} 2\right.$ compared to $\left.12.4 \mathrm{~cm}^{\wedge} 2\right)$. It has the completely recoverable dead volume under one milliliter and can accommodate volumes up to $50 \mathrm{~mL}$ if performing diafiltration in the same run, or up to $100 \mathrm{~mL}$ if only concentrating. The TFF system is capable of ultrafiltration rates up to 5 times faster compared to a $15 \mathrm{~mL}$ centrifugal device and is completely automated with non-contact liquid sensing, bringing the retentate volume down to the specified set point.

Baker Logan baker.logan@formulatrix.com

Dmitry Rodionov dmitry.rodionov@formulatrix.com

FORMULATRIX 